\title{
LIXISENATIDE IMPROVES GLYCEMIC CONTROL AND BODY COMPOSITION IN UNCONTROLLED TYPE 2 DIABETIC PATIENTS TREATED WITH INSULIN
}

Carral F, Ayala C, Piñero A, Jiménez Al, Expósito C, García C.

Endocrinology Department of Puerto Real University Hospital, Cádiz, Spain.

Objective: Evaluate the efficacy and safety of adding Lixisenatide to uncontrolled type 2 diabetic (T2DM) patients treated with insulin.

Methods: A prospective uncontrolled study was designed. Primary endpoints (measured at three and six months) were change in HbA1c, weight and insulin doses. Variables analyzed were: HbA1c level, insulin and other oral hypoglycemic agents (OHA) doses, capillary glucose tests, number and type of hypoglycaemias (glucose $<70 \mathrm{mg} / \mathrm{dl}$ ), side effects and body composition analysis (Tanita SC-330).

Results: Data from 42 T2DM patients (women: 55\%; mean age: $57.7 \pm 7.4$ years; T2DM duration: $13.5 \pm 8.7$ years; hypertension: $71 \%$; cardiovascular complications: $26 \%$ ) treated with insulin and lixixenatide were analyzed. Main results are expressed in tables and graphics.

Table 1. Changes in clinical, metabolic and treatment variables and hypoglycaemia during the study

\begin{tabular}{|c|c|c|c|c|}
\hline & Basal & 3 Months & 6 Months & $\mathbf{P}$ \\
\hline Weight (Kg) & $99.5 \pm 16.5$ & $96.2 \pm 14.4$ & $95.2 \pm 14.8$ & $<0.001^{1}$ \\
\hline Waist circumference (cm) & $118.0 \pm 12.3$ & $115.5 \pm 11.6$ & $114.9 \pm 14.2$ & $<0.001^{1}$ \\
\hline Body fat (\%) & $38.3 \pm 8.0$ & $38.5 \pm 8.4$ & $34.7 \pm 7.9$ & $0.547^{2} / 0.015^{3}$ \\
\hline HbA1c level (\%) & $9.2 \pm 1.7$ & $7.8 \pm 1.1$ & $7.6 \pm 0.8$ & $<0.001^{1}$ \\
\hline Insulin injections at day (n) & $2.3 \pm 1.3$ & $1.8 \pm 1.2$ & $1.9 \pm 1.3$ & $0.002^{2} / 0.047^{3}$ \\
\hline Patients with rapid insulin (n) & $19(46.3 \%)$ & $10(29.4 \%)$ & $7(29.2 \%)$ & $<0.001^{1}$ \\
\hline Basal insulin doses (UI) & $51.6 \pm 24.3$ & $45.3 \pm 24.7$ & $40.8 \pm 22.2$ & $0.062^{2} / 0.048^{3}$ \\
\hline Rapid insulin doses (UI) & $13.9 \pm 19.9$ & $9.3 \pm 15.5$ & $7.4 \pm 13.0$ & $0.002^{2} / 0.029^{3}$ \\
\hline Total insulin doses (UI) & $62.7 \pm 36.7$ & $53.6 \pm 35.6$ & $49.4 \pm 30.5$ & $0.055^{2} / 0.021^{3}$ \\
\hline Different type of OHA at day (n) & $0.9 \pm 0.9$ & $1.2 \pm 0.6$ & $1.2 \pm 0.6$ & $0.04^{2} / 0.119^{3}$ \\
\hline Capillary glucose test at day (n) & $1.8 \pm 0.9$ & $1.9 \pm 0.9$ & $1.6 \pm 0.9$ & $0.99^{2} / 0.102^{3}$ \\
\hline Hypoglycaemia episodes in last month (n) & - & $0.6 \pm 1.2$ & $1.0 \pm 1.7$ & - \\
\hline
\end{tabular}

${ }^{1}$ Three and six months vs basal; ${ }^{2}$ Three months vs basal; ${ }^{3}$ Six months vs basal.

Table 2. Patients leaving the study

\begin{tabular}{|l|c|}
\hline Reason for leaving the study & Results \\
\hline Nausea or vomiting (\%) & $4(9.5 \%)$ \\
\hline Hypertransaminasemia & $1(2.4 \%)$ \\
\hline Uncontrolled hyperglycemia & $1(2.4 \%)$ \\
\hline Unknown reason & $3(7.1 \%)$ \\
\hline Total & $9(21.4 \%)$ \\
\hline
\end{tabular}

Graphic 1. Change in main result variables at six months

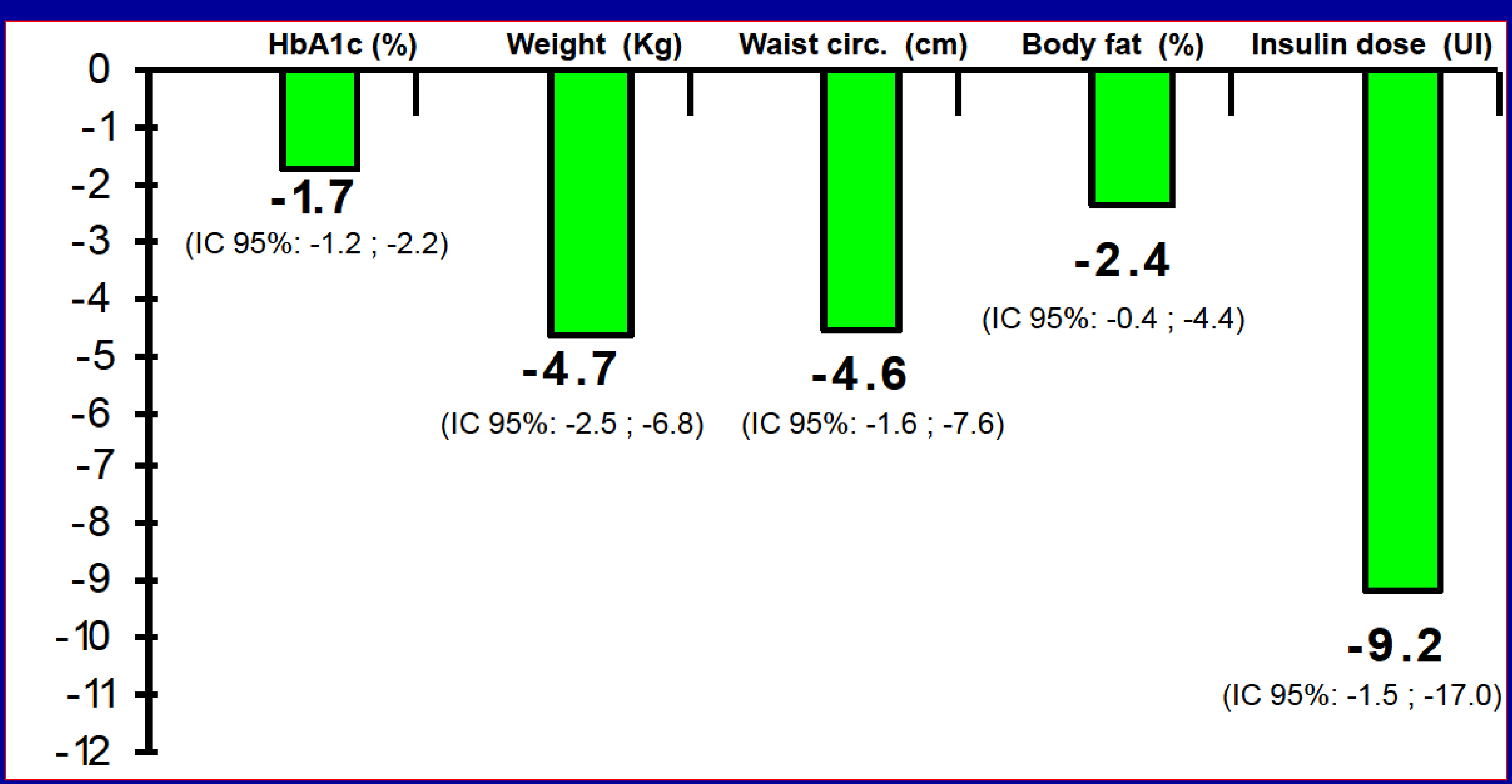

Conclusions: In our clinical experience, Lixisenatide contributes to improving glycemic control as facilitates weight loss and insulin doses reduction in T2DM patients uncontrolled with insulin. 